\title{
Genomic profiling using array comparative genomic hybridization define distinct subtypes of diffuse large b-cell lymphoma: a review of the literature
}

\author{
Carlos A Tirado ${ }^{1 *}$, Weina Chen ${ }^{2+}$, Rolando García ${ }^{3 \dagger}$, Kelly A Kohlman ${ }^{4+}$ and Nagesh Rao ${ }^{1}$
}

\begin{abstract}
Diffuse large B-cell lymphoma ( $\mathrm{DLBCL}$ ) is the most common type of non-Hodgkin Lymphoma comprising of greater than $30 \%$ of adult non-Hodgkin Lymphomas. DLBCL represents a diverse set of lymphomas, defined as diffuse proliferation of large B lymphoid cells. Numerous cytogenetic studies including karyotypes and fluorescent in situ hybridization (FISH), as well as morphological, biological, clinical, microarray and sequencing technologies have attempted to categorize DLBCL into morphological variants, molecular and immunophenotypic subgroups, as well as distinct disease entities. Despite such efforts, most lymphoma remains undistinguishable and falls into DLBCL, not otherwise specified (DLBCL-NOS). The advent of microarray-based studies (chromosome, RNA, gene expression, etc) has provided a plethora of high-resolution data that could potentially facilitate the finer classification of DLBCL. This review covers the microarray data currently published for DLBCL. We will focus on these types of data; 1) array

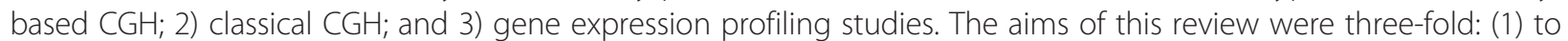
catalog chromosome loci that are present in at least $20 \%$ or more of distinct DLBCL subtypes; a detailed list of gains and losses for different subtypes was generated in a table form to illustrate specific chromosome loci affected in selected subtypes; (2) to determine common and distinct copy number alterations among the different subtypes and based on this information, characteristic and similar chromosome loci for the different subtypes were depicted in two separate chromosome ideograms; and, (3) to list re-classified subtypes and those that remained indistinguishable after review of the microarray data. To the best of our knowledge, this is the first effort to compile and review available literatures on microarray analysis data and their practical utility in classifying DLBCL subtypes. Although conventional cytogenetic methods such as Karyotypes and FISH have played a major role in classification schemes of lymphomas, better classification models are clearly needed to further understanding the biology, disease outcome and therapeutic management of DLBCL. In summary, microarray data reviewed here can provide better subtype specific classifications models for DLBCL.
\end{abstract}

Keywords: DLBCL, Array CGH, Genomic profiles

\footnotetext{
* Correspondence: ctirado@mednet.ucla.edu

${ }^{\dagger}$ Equal contributors

'Department of Pathology \& Laboratory Medicine UCLA - David Geffen UCLA, School of Medicine, Los Angeles, USA

Full list of author information is available at the end of the article
} 


\section{Introduction}

Diffuse large B-cell lymphoma (DLBCL) is the most frequent non-Hodgkin lymphoma comprising of greater than $30 \%$ of adult non-Hodgkin lymphomas in the West, and an even higher percent in developing countries [1]. DLBCL has traditionally been defined as a diffuse proliferation of lymphoid neoplasm in which the nucleus is equal to or exceeds the size of a normal macrophage nucleus [2]. It is clinically heterogeneous and includes a wide spectrum of lymphoid neoplasms.

Many morphological, biological, cytogenetics and clinical studies have attempted to subdivide DLBCL into morphological variants, molecular, immunophenotypic subgroups, and distinct disease entities. While progress has been made in the recent years, many of the cases remain biologically heterogeneous [3,4]. In fact, the most recent World Health Organization (WHO) classification published in 2008 groups most of these lymphomas into the category of DLBCL, not otherwise specified (DLBCL-NOS) [4].

One recent microarray technology applied to distinguish between DLBCL subtypes is array based comparative genomic hybridization (aCGH). Array-based CGH provides high resolution genome wide measurement of DNA copy number alterations highlighting patterns of deletions and amplifications. An additional advantage of array-based CGH is the assessment of allelic ratio or loss of heterozygosity. Here, an assessment of the relative intensity for each of the alleles can be determined. However; one limitation is the inability to detect balanced chromosome translocations. Nonetheless, aCGH can facilitate new construct classifiers for DLBCL subtypes. Other key technology worth mentioning that may provide classification models for DLBCL include single nucleotide polymorphism (SNP) arrays. Indeed, in recent years a number of SNP array studies have been reported that list characteristic SNP in hematolymphoid neoplasms [5-9].

An alternative microarray technology that has been extensively used in the classification of DLBCL is gene expression profiling. Studies using gene expression profiling have stratified DLBCL into favorable and unfavorable groups i.e., the germinal center B-cell like (GCB) and the activated $\mathrm{B}$-cell like (ABC) DLBCL respectively $[1,10]$. The $A B C$ type expresses genes that are distinctive of activated B-cells and plasma cells with a poor clinical outcome (30\% 5-year survival rate), whereas the GCB subtype expresses a molecular signature of normal germinal center B-cells with a more favorable overall survival (59\% 5-year survival rate) [11-14]. Amplifications of the $R E L$ loci, $B C L-2$ translocations and hypermutations of the immunoglobulins loci are typical of the GCBDLBCL subtype $[1,11,15]$. In contrast, constitutive activation of the nuclear factor $k B$ pathway is a distinctive feature of both the $\mathrm{ABC}$ and primary mediastinal B-cell lymphoma (PMBL) subtype [16-19]. A third type also identified from others by molecular profiling is PMBL with frequent amplifications at $2 \mathrm{p}$ and $9 \mathrm{p}$ corresponding to $J A K-2$ and $R E L$ respectively with a $64 \% 5$-year survival rate $[2,11,13,20,21]$. Further studies with high resolution array comparative genomic hybridization $(\mathrm{aCGH})$ have revealed recurrent copy number alterations (CNA), as well as prognostic indicators in a number of DLBCL subtypes [22-27], for example, in a recent high resolution CGH study, CNA resistant to rituximab, cyclophosphamide, doxorubicin, vincristine, and prednisone (R-CHOP) therapy included amplifications of $1 \mathrm{p} 36.13$, 1q42.3, 3p21.31, 7q11.23 and 16p13.3, as well as losses at $9 \mathrm{p} 21.3$ and $14 \mathrm{p} 21.31$ in DLBCL. Various reasons have been proposed for the various CNA just mentioned above and immuno-chemoresistance. These include: faulty p53/INK/ARF functioning caused by 9p21.3 deletions seems to disrupt p53 induced apoptosis, up regulation of various target genes in the nuclear factor $k B$ pathway due to copy number gain of $M A P K A P K 3$ at $3 \mathrm{p} 21.31$ leading to nuclear factor $k B$ activation and consequently resulting in high expression of various apoptotic inhibiting genes [28-31] and copy number gains at $16 \mathrm{p} 13.3$ resulting in overexpression of $A B C A 3$, which has been implicated as a likely cause of drug resistance by driving the flow of drugs out of the cell [32].

Despite these efforts to subdivide DLBCL, the relationship between the different classification schemes has not been adequately studied. In this review, we explored CNA linked to well-defined WHO subtypes of DLBCL and compared CNA across the various DLBCL subtypes.

\section{Review of the literature}

\section{Diffuse large B-cell lymphoma, not otherwise specified} (NOS)

DLBCL NOS consists of all DLBCL cases that do not fit into one of the other specific subtypes or disease entities [1]. Since the NOS subgroup continues to exist as an indistinct set of DLBCL, the exact associated aberrations are therefore more difficult to define. A study carried out by Pasqualucci et al. [33] revealed aberrant somatic hypermutations targeting multiple genetic loci including PIMI, MYC, RHOH/TTF, and PAX. Moreover, abnormalities of band region $3 \mathrm{q} 27, \mathrm{t}(14 ; 18)$ translocations and complex karyotypes are commonly seen in this subtype.

\section{Array CGH and gene expression profiles identify molecular subtypes}

In 2008, Lenz et al. [2] analyzed 203 DLBCL samples by high resolution array $\mathrm{CGH}$ and gene expression profile to investigate the 3 molecular subtypes of DLBCL. Aberrations most characteristic of $\mathrm{ABC}$ DLBCL included 
trisomy 3, deletion of chromosome arm 6q, deletions/ duplications of 18q, deletion of INK4a/ARF tumor suppressor locus on chromosome 9, and gain-amplification of a 9-megabite $(\mathrm{Mb})$ region on chromosome 19q. Trisomy 3 was the most frequent aberration seen in $\mathrm{ABC}$ DLBCL (26\%). In cases with trisomy 3, FOXP1 was the most frequently up-regulated gene. Indeed, high FOXP1 expression is a characteristic finding of the ABC DLBCL subtype and FOXP1 has been associated as an oncogene [12,34,35]. In total, $38 \%$ of ABC DLBCL had an increase in FOXP1 CNA compared to $4 \%$ and $3 \%$ for GCB DLBCL and PMBL each. Another subtype specific lesion for $A B C$ DLBCL included NFKBIZ identified in a chromosome 3 amplicon. It was detected in $9 \%$ of the cases, while never identified in GCB DLBCL or PMBL. $N F K B I Z$ activates targets in the nuclear factor $k B$ pathway a hallmark for ABC DLBCL [36]. In terms of the gain/amplification of $18 \mathrm{q}$, which was significantly more frequent in $\mathrm{ABC}$ DLBCL than the other molecular subtypes, BCL-2 and NFATC1 were consistently upregulated by the gain/amplification of 18q. Another distinctive feature of ABC DLBCL compared to the other two molecular subtypes was deletion of the INK4a/ARF tumor suppressor locus; $30 \%$ of the ABC DLBCL cases were deleted compared to $4 \%$ in GCB DLBCL and $6 \%$ in PMBL. This locus encodes for three tumor suppressors: CDKN2A (p16), CDKN2B (p15) and p14 ARF. For gain/ amplification of $19 \mathrm{q}$, the overexpression of the SPIB gene seems to play a more functional role in the pathogenesis of ABC DLBCL. Recent work has revealed a translocation between SPIB and the immunoglobulin heavy chain at 14q32 in ABC DLBCL [37]. Aberrations seen in GCB DLBCL included amplification of the mir-17-92 microRNA in the MIHG1 locus cluster on chromosome 13, which has been shown to collaborate with $M Y C$ to transform B-cells and to reduce apoptotic activity [38]. The 1.4-Mb amplified region on chromosome 13 was detected $12.5 \%$ of the time in GCB DLBCL, rarely in PMBL (3\%), and not observed in ABC DLBCL. A gain of a 7.6-Mb region on chromosome 12 revealed upregulation of $M D M 2$, a negative regulator for the p53, while deletion of the PTEN tumor suppressor gene on chromosome 10, and amplification of the REL locus on chromosome 2 were also more common in this molecular subtype. Interestingly, cases with PTEN deletion had a $\mathrm{t}(14 ; 18)$ translocation suggesting that loss of PTEN may play a significant role in the pathogenesis of GCB DLBCL with $t(14 ; 18)$. The most frequent chromosomal lesions seen in PMBL included amplifications of a telomeric region of chromosome 9p, monosomy 10, and gain/amplification of chromosome 20p. It is unclear what genes in these regions are functionally important in the pathogenesis of PMBL. Of note, genes that were more frequently up-regulated in these regions were $J A K-2$ and T-cell inhibitor ligand $P D-L 2$. These results confirm that DLBCL can be confidently sub-grouped based on array CGH results and gene expression profiles. In another study by Tagawa et al. [22], array CGH was used to further study the aberrations associated with $A B C$ DLBCL and GBC DLBCL. Based on their results, the ABC DLBCL group was genomically characterized by more frequent gains of 3q23-q28, 18q11.2-q23, 19q13.41q13.43 and loss of 6q22.31-q24.1 and 9p21.3, while the GCB group was genomically characterized by more frequent gains of 1q21.1-q23.3, 1q31.1-q42.13, 2p15-p16.1, 7q22.1-q36.2, and 12q13.1-q14. These results suggest that ABC DLBCL and GCB DLBCL are genetically distinct from one another and arise from separate genetic pathways [2] Table 1.

\section{Large B-cell lymphoma specified by site Primary mediastinal large B-cell lymphoma}

Primary Mediastinal Large B-cell Lymphoma (PMBL) arises in the thymus from thymic B-cells that presents as a mass in the mediastinum [1] and represent a distinct entity within the germinal center (GC) derived high grade DLBCL [39]. Although classified as a subtype of DLBCL, it is noteworthy to mention that PMBL and classical Hodgkin lymphoma (HL) share remarkably similar molecular profiles, as well as certain clinical and histological features $[19,40]$. In efforts to better define PMBL, Palanisamy et al. [39] using classical CGH characterized PMBL by whole chromosome gains of 12, 21, 22 and whole chromosome loses of 11, 13, and 18. Also, frequent gains of 4q, 9q, 10p, 17p, 19p, 20q, 21q, 22q and losses of 3q, 7p, 8q, 9p, 11p, 11q, 13q, 18p, 18q, Xp, and $\mathrm{Xq}$ were seen in PMBL patients. Subsequent studies used array based CGH to document amplification of

Table 1 Genomic Gains and Losses in ABC and GCBDLBCL Molecular Subtypes

\begin{tabular}{|c|c|c|c|}
\hline Chromosome breakpoints & GCB DLBCL & ABC-DLBCL & Reference \\
\hline $1 q 21.1-q 23.3 \mathrm{G}$ & G & & 16 \\
\hline $1 q 31.1-q 42.13$ & G & & 16 \\
\hline $2 p 15-16.1$ & G & & 16 \\
\hline 3 & & G & 16 \\
\hline $3 q 23-q 28$ & & G & 16 \\
\hline $6 q$ & & L & 16 \\
\hline $6 q 22.31-q 24.1$ & & $L$ & 2 \\
\hline $7 q 22.1-q 36.2$ & G & & 16 \\
\hline $9 p 21.3$ & & $L$ & 16 \\
\hline $12 q 13-q 14.1$ & G & & 16 \\
\hline $18 q$ & & G & 16 \\
\hline 19q13.41-q13.42 & & G & 16 \\
\hline
\end{tabular}

Key: G, genomic gain; L, loss of genetic material. 
band region 9p24.1 in PMBL cell lines [40], and similar studies reported amplifications to $9 \mathrm{p}$ and $2 \mathrm{p}$ involving the REL locus on PMBL [21,40-42]. In 2007, Wessendorf et al. [43] further outlined chromosomal aberrations in PMBL using aCGH $(\mathrm{n}=37)$. Here, genomic gains of 9p24 (68\%), 2p15 (51\%), 7q22 (32\%), 9q34 (32\%), 12q (30\%) and 18q21 (22\%) were reported. Interestingly, this study also described 17 chromosome regions with genomic losses in more than $10 \%$ of the cases. In terms of clinical outcome, PMBL is now more favorable due to intensive chemotherapy and radiation therapy [44]. As stated previously, PMBL and cHD share very similar molecular profiles, for example, frequent amplifications at 9p24 have been reported in both PMBL and classical HL; however, it is of interest to note that a study by Feys and colleagues using cHD cell lines [45] reported deletions of chromosome 15q26.2 encompassing RGMA and CHD2, STAT6 up-regulation and deletion at 16q12.1 that were not found to occur in this current review of PMBL. Further investigation is needed to see whether these regions can be used to distinguish PMBL from cHD.

\section{Large B-cell lymphoma of the bone (not listed as an entity in 2008 WHO)}

Large B-cell lymphoma of the bone (LBCLB) is a subtype of primary extra nodal DLBCL. It typically presents in the longer bones, such as the humerus, tibia, pelvis, spine or the femur. Patients present with pain, a palpable mass or fractures. Complete remission is frequently obtained with a combination of chemotherapy and radiotherapy $[9,46]$. In 2010, Heyning et al. [46] used array-CGH to study nine primary lymphoma of the bone. Aberrations that were frequently seen included loss of 1p35-36.3, 6q14-27, 14q32, 15q11-26, trisomy 7, gain of 1q21-44, 6p21 and amplification of 2p16.1. Eight of nine patients reached complete remission in this study. These results support previously described GClike properties of LBCLB, particularly that of better clinical outcome, 1q gain and 2p16.1 amplifications.

\section{$D L B C L$ of the central nervous system}

DLBCL of the CNS represents a subtype that comprises all primary intracerebral or intraocular lymphomas [1]. Booman et al. reported genomic aberrations associated with DLBCL of the CNS using an array-based CGH $(n=9)$. The most common genomic aberrations seen in this study were loss of 6p21.32-p25.2 (56\%), 6q (56\%), $17 \mathrm{p} 12-\mathrm{p} 13.3$ (56\%) and gains of 1q21.3-q32.1 (33\%), 12(44\%), 15q12-q21.1 (22\%), 7/7q (22\%), 18q (22\%) and $19 \mathrm{q} 13.12-\mathrm{q} 13.43$ (22\%) [47]. A similar study by Montesinos et al. [48] reported similar findings. DLBCL of the CNS is predominately of the molecular $A B C$ subtype, thus explaining the poor prognosis of this subset of DLBCL [49].

\section{Primary cutaneous $L B C L$, leg type}

Based on the WHO classification scheme, there are three types of primary cutaneous B-cell Lymphoma: leg type (PCBCL), follicular center, and marginal zone. Leg type is a DLBCL that presents with large transformed Bcells that commonly arise in the leg at first [1]. In One study [50], using array $\mathrm{CGH}$ and fluorescent in-situ hybridization on 6 well characterized cases of PCBCL reported distinct genomic aberrations. This study showed recurrent gains of $1 \mathrm{p} 36.33,3 \mathrm{p} 21.3,7 \mathrm{p}, 7 \mathrm{q} 11.21$, 7q21.1, 11q13, 12q12-q13, 17q11.2, 17q21-22, 18q11.2, $18 \mathrm{q} 21.1,19 \mathrm{q}$ and losses to $9 \mathrm{p} 21,6 \mathrm{q} 22-\mathrm{q} 23$ and $17 \mathrm{p} 11.2-$ p12 in $50 \%$ or more of the cases. Among the chromosome regions with recurrent gains found in at least 33\% of the cases included: 1q25-q31, 1q41, 1q, 2p22, 2p12q11, 3p21-p25, 3q, 7q, 8q24, 9p12-q21, 11q, 12p11, $12 \mathrm{q} 13-\mathrm{q} 15,12 \mathrm{q} 32,16 \mathrm{q} 23,17 \mathrm{q} 11-\mathrm{q} 12,17 \mathrm{q} 21,18 \mathrm{q}$, $19 q 13,20 q 13,22 q 11$ and 22q13. Similarly, recurrent losses in $33 \%$ of the cases were $1 \mathrm{p} 36.31,1 \mathrm{p} 31-\mathrm{p} 32,1 \mathrm{p} 13$, 4q, 6q, 8p, 8q11, 9p11, 14q, Xq13 and Xq25. One of the most frequent aberrations mentioned in this study was the loss of 9p21(83\%). Of the six patients with 9p21 deletion, all died in this study. Similar observations have reported deletions to $9 \mathrm{p} 21.3$, for example, one study identified 9p21 loss in eight of 12 patients with PCBCL [51]. Of the seven patients that died in this study, five cases had a deletion at $9 \mathrm{p} 21$. This may suggests that loss of this region has important clinical significance. In a recent study, it was revealed that the incidence of $9 \mathrm{p} 21$ loss was more prevalent in the $\mathrm{ABC}$ molecular subtype compared to the GC DLBCL subtype, demonstrating a poor clinical outcome for the loss of 9p21 [23]. Indeed, PCBCL is associated with the ABC DLBCL molecular subtype and requires therapy intensification [50].

\section{Large B-cell lymphoma specified by histology, phenotype, or genotype \\ T-cell Histiocyte-rich B-cell lymphoma}

T-cell/histiocyte-rich B-cell lymphoma (T/HR) has been categorized as a DLBCL, but it had been done with much controversy due to the ambiguous presentation of the malignancy. T/HR LBCL is distinguished by a few scattered, large, atypical B-cells surrounded by a large quantity of T-cells and scattered histiocytes [1]. Classical CGH studies by Franke et al. [52] revealed most common gains of 4q13q38,18q21, Xq and Xp21-p11, as well as recurrent losses $17 \mathrm{p}$. Most frequent $\mathrm{CNA}$ in this above report were Xq12-13 (58\%), 4q25-q26 (41\%), Xp11-21 (29\%), 18q21 and 17p (24\% each). Molecular profiles have identified most T/HR DLBCL cases to a subgroup of DLBCL distinguished by a "host response" 
with an adverse clinical outcome [53]. To the best of our knowledge, there is no array based CGH reports on this entity.

\section{De novo, CD5+ large B-cell lymphoma}

DLBCL expresses a variety of B-cell surface markers including CD5 in approximately $10 \%$ of cases [54]. Previous studies have reported a worst clinical outcome for CD5+ DLBCL compared to CD5- DLBCL $[55,56]$. In a genome wide array based CGH $(n=25)$, Tagawa et al. [57] identified genomic gains in more than $30 \%$ of the case in the following band regions: 1q23.1, 3q12.1, 3q27.3, 11q23.3, 11q24.3, 12p12.1, 12q13.3, 12q14.1, 12q15, 13q21.32, 13q32.3, 16p13.3, 18q21.1, 18q22.3, 19q13.33, 19q13.41, 19q13.43. Likewise, genomic losses were detected in 1p36.32, 6q21, 8p23.3, 9p21.3 and 17p13.1. Of note, by concentrating on the gain of 13q21-34 and loss of 1p34-36, Tagawa et al. was also able to recognize prognostically distinct subgroups within the CD5+ DLBCL subset. Moreover, in a separate study [23], Tagawa et al. identified 3q23-3q28 (31\%), 6q22.31-q24.1 (44\%) and 9p21.3 (50\%) in high frequency for CD5+ DLBCL. When comparing CNA and clinical outcome, this latter study demonstrated 9p21 marked the most aggressive cases. In fact, Kreisel et al. [27] showed 9p21.3 band region as chemoresistant in DLBCL.

\section{Large B-cell lymphoma (LBCL) associated with epstein-} barr virus and/or kaposi sarcoma-associated herpesvirus/ human herpesvirus 8

$D L B C L$ associated with chronic inflammation

Diffuse large B-cell lymphoma associated with chronic inflammation $(\mathrm{CI})$ is a DLBCL associated with long lasting inflammation that is associated with $\mathrm{EBV}+$. In most cases, this subtype of DLBCL develops in small body cavities and narrow spaces $[1,10]$.

\section{Pyothorax-associated lymphoma}

Pyothorax-associated Lymphoma (PAL) develops in the pleural cavity of patients with a history of long-standing pyothorax [1]. Most reported cases of PAL have occurred in Japan, with few cases reported in western countries. Immunoglobulin genes in PAL are usually clonally rearranged and usually TP53 mutations can be seen $[58,59]$. PAL is usually a precursor to DLBCL associated with chronic inflammation [10]. Few studies have attempted to define distinct CGH profiles for the PAL subtype. Using classical CGH analysis, Yamato et al. [60] showed the amplification of the 8q24 band region in 7 PAL cases. Amplification was later confirmed in four cases by southern blot analysis. Another separate study reported an over-expression of the interferon alpha- inducible protein 27, IFI27 [61]. By cytogenetic analysis, one study reported complex karyotypes but no common abnormality was reported [62].

\section{Plasmablastic lymphoma}

Plasmablastic lymphoma (PL) is a scattered proliferation of large neoplastic cells that have the immunophenotype of plasma cells but resemble B-immunoblasts. It was originally described in the oral cavity but may occur in other extra-nodal sites [1]. In 2009, an array-based CGH conducted by Chang et al. [63] reported gains ( $>40 \%)$ of 1p36.11-1p36.33, 1p34.1-1p36.13, 1q21.1-1q23.1, 7p21.37p23 (38\%), 7q11.2-7q11.23, 8q24.3 (25\%), 10p12 (23\%), 11q12-11q13.2, 14q32 (31\%), 16p13.2-p13.3 (38\%), 16q24 (38\%), 17p13 (38\%), 20q11.1-q11.23 (38\%) and 22q12.2-22q13.3 in PL, while genetic losses were more diverse. However, only 1p35.1-1p36.12, 1q21.1-1q23.1 and $1 \mathrm{p} 36.11-1 \mathrm{p} 36.33$ were unique to PL when compared with DLBCL (AIDS related and non-AIDS related) and plasma cell myeloma. The clinical outcome for these patients is poor [64].

\section{Primary effusion lymphoma}

Primary Effusion Lymphoma (PEL) is another rare subtype of DLBCL that presents as an extravascular collection of fluids with no identifiable tumor mass. It is associated with the Human Herpes Virus 8 (HHV8 +), the Kaposi sarcoma herpes virus (KSHV) and carries a poor clinical outcome in PEL patients $[1,65,66]$. Very little is known about the genetic aberrations associated with this malignancy. A recent array CGH study revealed gains of 1q21-41 (47\%), 4q28.3-35 (29\%), 7q (58\%), 8q (63\%), 11 (32\%), 12 (61\%), 17q (29\%), 19p (34\%), 20q (34\%) and losses of 4q (32\%), 11q25 (29\%) and 14q32 (63\%) [67]. In an earlier study using classical CGH $(\mathrm{n}=5)$, Ohshima et al. [68] identified gains of 3q13-q27, 8q24, 8, 10q21-23 and Yq in HHV8 negative PEL cases.

\section{Unclassifiable types}

$\angle B C L$ with features intermediate between DLBCL and Burkitt lymphoma

Large B-cell lymphoma with features intermediate between DLBCL and Burkitt lymphoma (INT) is an aggressive lymphoma that has overlapping features between DLBCL and Burkitt lymphoma. Approximately 35-50\% of INT cases have $M Y C$ rearrangements [69,70] with a concurrent $B C L-2$ translocation ("double-hit lymphoma") in approximately $15 \%$ of cases. A limited number of array based CGH studies are available. In 2006, Hummel et al. [69] using aCGH reported most of these cases with a high chromosomal complexity with a score of 6 or more abnormalities with a $M Y C$ rearrangement. 
Table 2 Array CGH data of gains and losses for the various DLBCL Subtypes

\begin{tabular}{|c|c|c|c|c|c|c|c|c|c|}
\hline Chromosome breakpoints & PMBL & Bone & CNS & Leg type & T/HR & CD5+ & PAL & $\mathrm{PL}$ & PEL \\
\hline $1 p 36$ & & - & & $+/-$ & & - & & + & \\
\hline 1p35-p34 & & - & & & & & & + & \\
\hline $1 q$ & & & & + & & & & & \\
\hline $1 q 11$ & & & & + & & & & & \\
\hline $1 q 21$ & & + & + & + & & & & + & + \\
\hline $1 q 22$ & & + & + & + & & & & + & + \\
\hline $1 q 23$ & & + & + & + & & + & & + & + \\
\hline $1 q 24-q 25$ & & + & + & + & & & & & + \\
\hline $1 q 31-q 32$ & & + & + & + & & & & & + \\
\hline $1 q 36$ & & + & & + & & & & & + \\
\hline $1 q 41$ & & + & & + & & & & & + \\
\hline $1 q 43-q 44$ & & + & & + & & & & & \\
\hline \multicolumn{10}{|l|}{2} \\
\hline $2 p 22$ & & & & + & & & & & \\
\hline $2 \mathrm{p} 16$ & & + & & & & & & & \\
\hline $2 p 15$ & + & & & & & & & & \\
\hline $2 p 12$ & & & & + & & & & & \\
\hline \multicolumn{10}{|l|}{$2 \mathrm{p} 13$} \\
\hline \multicolumn{10}{|l|}{$2 \mathrm{p} 11$} \\
\hline \multicolumn{10}{|l|}{$2 q 33$} \\
\hline \multicolumn{10}{|l|}{3} \\
\hline 3p21-p25 & & & & + & & & & & \\
\hline $3 p 21$ & & & & + & & & & & \\
\hline \multicolumn{10}{|l|}{$3 p 14$} \\
\hline $3 q$ & & & & + & & & & & \\
\hline $3 q 12-q 24$ & & & & & & + & & & \\
\hline $3 q 25$ & & & & & & + & & & \\
\hline $3 q 26-q 27$ & & & & & & + & & & \\
\hline $4 q 28-35$ & & & & & & & & & + \\
\hline $4 q$ & & & & - & & & & & - \\
\hline \multicolumn{10}{|l|}{5} \\
\hline \multicolumn{10}{|l|}{$5 p 15$} \\
\hline \multicolumn{10}{|l|}{$5 p 13$} \\
\hline \multicolumn{10}{|l|}{$5 q 11-q 31$} \\
\hline \multicolumn{10}{|l|}{6} \\
\hline 6p25-p22 & & & - & & & & & & \\
\hline $6 \mathrm{p} 21$ & & + & - & & & & & & \\
\hline $6 q$ & & & - & - & & & & & \\
\hline $6 q 14-q 21$ & & - & - & - & & - & & & \\
\hline $6 q 22$ & & - & & - & & - & & & \\
\hline $6 q 23-q 27$ & & - & & - & & & & & \\
\hline 7 & & + & + & . & & & & & \\
\hline $7 p$ & & & & + & & & & & \\
\hline $7 p 21$ & & & & + & & & & + & \\
\hline $7 q$ & & & + & & & & & & + \\
\hline $7 q 11$ & & & + & + & & & & + & + \\
\hline $7 q 21$ & & & + & + & & & & & + \\
\hline
\end{tabular}


Table 2 Array CGH data of gains and losses for the various DLBCL Subtypes (Continued)

\begin{tabular}{|c|c|c|c|c|c|c|c|}
\hline $7 q 22$ & + & & + & + & & & + \\
\hline $7 q 31$ & & & + & + & & & + \\
\hline $7 q 32$ & & & + & + & & & + \\
\hline \multicolumn{8}{|l|}{8} \\
\hline $8 p$ & & & & - & & & \\
\hline $8 p 11$ & & & & - & & & \\
\hline 8p23-p21 & & & & - & - & & \\
\hline $8 q$ & & & & & & & + \\
\hline $8 q 24$ & & & & + & + & + & + \\
\hline \multicolumn{8}{|l|}{9} \\
\hline $9 p 11$ & & & & - & & & \\
\hline $9 p 24$ & + & & & & & & \\
\hline $9 p 12-21$ & & & & + & & & \\
\hline $9 p 21$ & & & & - & - & & \\
\hline $9 q 34$ & + & & & & & & \\
\hline \multicolumn{8}{|l|}{$10 q$} \\
\hline \multicolumn{8}{|l|}{$10 p$} \\
\hline $10 \mathrm{p} 12$ & & & & & & + & \\
\hline \multicolumn{8}{|l|}{$10 q 21-q 23$} \\
\hline 11 & & & & & & & + \\
\hline \multicolumn{8}{|l|}{$11 p$} \\
\hline $11 q$ & & & & + & & & \\
\hline $11 q 12$ & & & & & & + & \\
\hline $11 q 13$ & & & & + & & + & \\
\hline \multicolumn{8}{|l|}{$11 q 21-22$} \\
\hline $11 q 23-24$ & & & & & + & & \\
\hline $11 q 24-q 25$ & & & & & & & - \\
\hline 12 & & & + & & & & + \\
\hline $12 \mathrm{p} 11-12$ & & & & + & + & & \\
\hline $12 q$ & + & & & & & & \\
\hline $12 q 12$ & + & & & + & & & \\
\hline $12 q 13-q 23$ & + & & & + & + & & \\
\hline $12 q 24$ & + & & & & & & \\
\hline \multicolumn{8}{|l|}{13} \\
\hline \multicolumn{8}{|l|}{$13 p 11$} \\
\hline \multicolumn{8}{|l|}{$13 q$} \\
\hline $13 q 21-q 31$ & & & & & + & & \\
\hline $13 q 32$ & & & & & + & & \\
\hline $13 q 33 q 34$ & & & & & + & & \\
\hline \multicolumn{8}{|l|}{14} \\
\hline $14 q$ & & & & - & & & \\
\hline $14 q 32$ & & - & & - & & + & - \\
\hline \multicolumn{8}{|l|}{15} \\
\hline \multicolumn{8}{|l|}{$15 \mathrm{p} 11$} \\
\hline $15 q$ & & - & & & & & \\
\hline $15 q 12$ & & - & - & & & & \\
\hline $15 q 13-q 14$ & & - & - & & & & \\
\hline
\end{tabular}


Table 2 Array CGH data of gains and losses for the various DLBCL Subtypes (Continued)

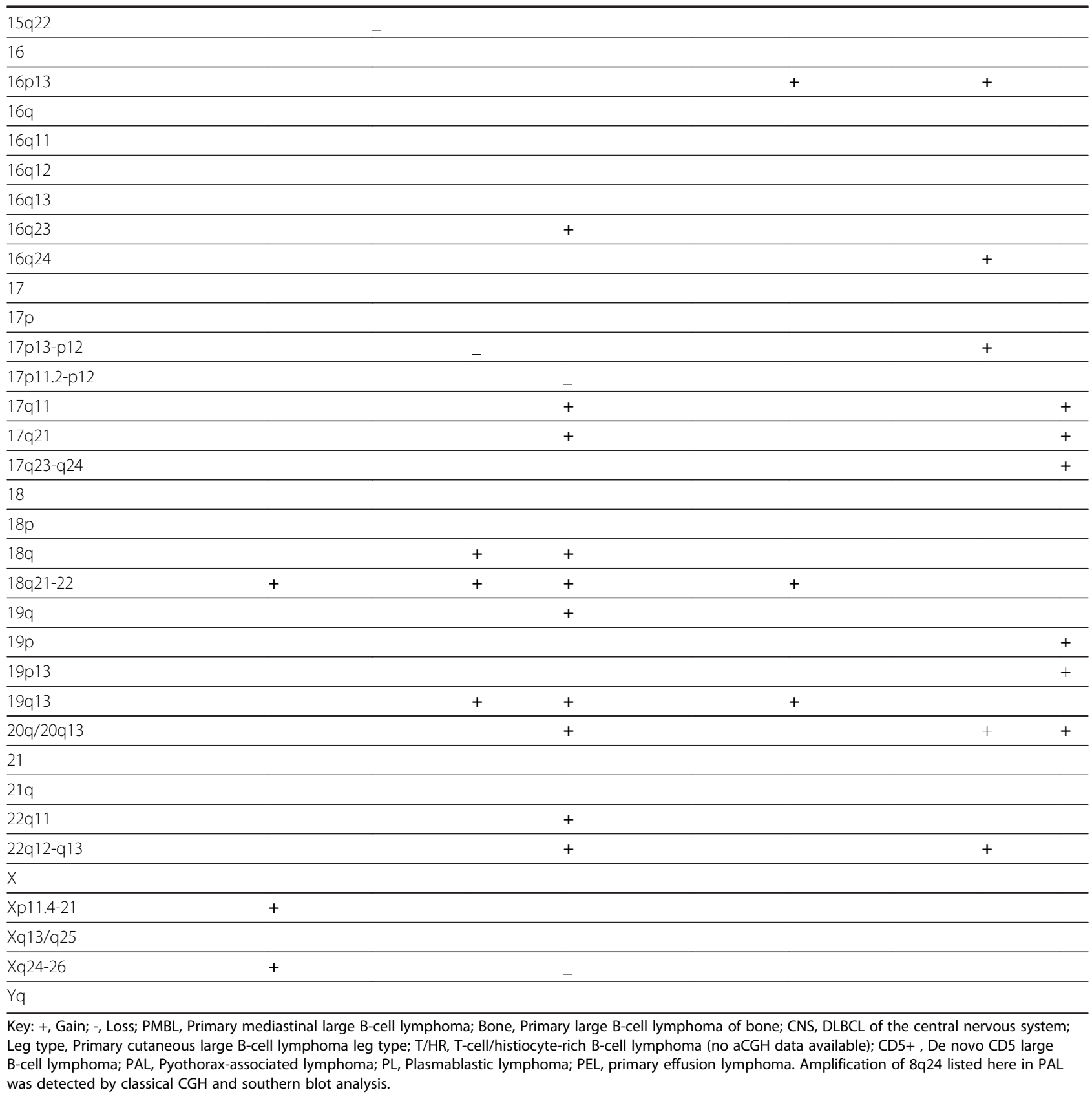

\section{DLBCL subtypes with limited or no array-based CGH information \\ Intravascular large B-cell lymphoma}

Intravascular Large B-cell lymphoma (ILBCL) is differentiated by localization of atypical lymphomatous cells within smaller vessels and capillaries $[1,71]$. It is a rare, aggressive extra nodal B-cell lymphoma that is recognized in the 2008 WHO classification as a subset of DLBCL. ILBCL is known as the great imitator due to the fact that it presents with a broad spectrum of nonspecific symptoms with no distinct array CGH profile [10].

\section{Anaplastic lymphoma kinase-positive $L B C L$}

Anaplastic large cell lymphoma with expression of the anaplastic kinase protein is a rare neoplasm of ALKpositive monomorphic large B-cells with immunoblastic or plasmablastic morphology. The only chromosome aberration associated with this disorder is the involvement of the $A L K$ gene on chromosome 2. The gene is usually involved with the following translocations: $t(2 ; 17)(\mathrm{p} 23$; $\mathrm{q} 23)$ or $\mathrm{t}(2: 5)(\mathrm{p} 23 ; \mathrm{q} 35)$. This type of lymphoma is a rare entity constituting only less than $1 \%$ of DLBCL cases. Thus, no substantial CGH data is available $[1,10]$. Patients 
are unresponsive to rituximab and have a poor clinical outcome [72].

\section{$E B V+D L B C L$ of the elderly}

Epstein-Barr virus-positive DLBCL of the elderly is a newly recognized subtype of DLBCL by the WHO classification (2008) which presents as an EBV + clonal B-cell proliferation usually in patients over 50 years $[1,73,74]$. $\mathrm{EBV}+\mathrm{DLBCL}$ is such a new classification of DLBCL that there is not sufficient $\mathrm{CGH}$ array data to create a genomic profile or distinctive cytogenetic pattern for such malignancy [75].

\section{Large B-cell lymphoma arising in HHV8+ multicentric castleman disease}

LBCL occurring in HHV8+ associated multicentric castleman disease is characterized by monoclonal proliferation of HHV8+ lymphoid cells in the presence of multicentric castleman disease [1]. It is an aggressive disorder with no information about the cytogenetics or CGH profiles $[1,76]$.

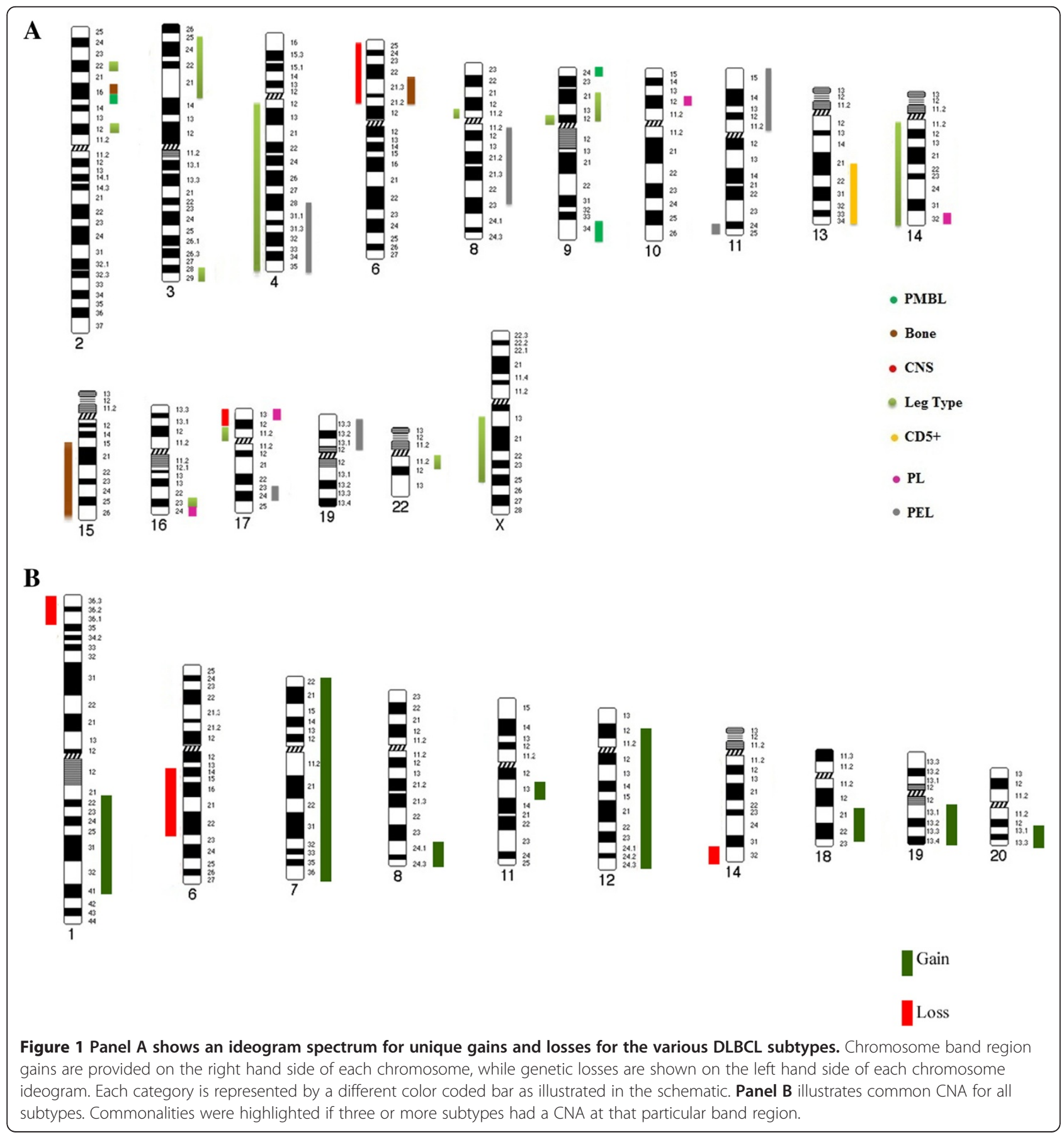




\section{Lymphomatoid granulomatosis}

Lymphomatoid Granulomatosis (LG) is an angiocentric and angiodestructive proliferative B-cell neoplasm that involves extra-nodal sites. LG is composed of malignant B-cells that are Epstein-Barr virus-positive combined with reactive T-cells. Clinical outcome is variable and depended on the quantity of large B-cells [1]. The majority of studies on LG have failed to identify unique chromosomal aberrations; however, a study by GoddeSalz et al. [77] reported few consistent chromosome aberrations including trisomy of chromosome 3 and 5 along with duplications of the $\mathrm{X}$ chromosome.

\section{$\angle B C L$ with features intermediate between $D L B C L$ and Hodgkin lymphoma}

This type of lymphoma is characterized by clinical and morphological features that overlap between DLBCL, particularly primary mediastinal large B-cell lymphoma, and classic Hodgkin lymphoma. Only limited cases of LBCL with features intermediate between DLBCL and Hodgkin lymphoma have been studied.

The table below summarizes genomic gains and losses for the various DLBCL subtypes, Table 2-, Figure 1-.

\section{Conclusions}

In this review, we explored associated copy number alterations (CNA) in 2008 WHO-defined DLBCL subtypes by array $\mathrm{CGH}$. Certain chromosomal aberrations that were significantly more frequent in a particular DLBCL subtype than in the others, and some of these aberrations were associated with clinical outcome. Following our review of aCGH microarray studies, a number of subsets were re-classified, for example, unique chromosome loci were identified in the following subtypes: PMBL, gain at 2p15, 9p24, 9q34, Xp11.4-21, Xq24-26; Large B-cell lymphoma of the bone, gain at 2p16, 6p21 and loss at 15q15-q26; DLBCL of the CNS, loss at 6p21-25,17p12-13; Leg type DLBCL, gain at 2p22, 2p12, 3p21-25, 3q28-29, 9p12-21,16q23,22q11and loss at 4q, 8p11,14q, Xq13-25; CD5+ DLBCL, gain at 13q21-34; Plasmablastic lymphoma, gain at 10p12, $14 \mathrm{q} 32,16 \mathrm{q} 24,17 \mathrm{p} 12-13$ and primary effusion lymphoma with gains at 4q28-35, 8q11.2-23.1, 11p, 17q23-24, 19 p13 and loss at 11q24-25. However, despite these efforts, there is still a number of unclassifiable DLBCL subtypes post-microarray studies. Among these include intravascular LBCL, EBV + DLBCL of the elderly, large B-cell lymphoma arising in HHV8+ multicentric Castleman disease, Lymphomatoid granulomatosis, LBCL with features intermediate between DLBCL and HL and PAL. Several reasons for this may include: a limited number of study cases, uncommon disease entities and a comparatively small number of publicly available aCGH datasets. Therefore, future studies should aim on the copy number alterations in newly defined uncommon large B-cell lymphoma entities, such as EBV + DLBCL of the elderly, ALK positive DLBCL and LBCL arising in HHV8-associated multicentric castleman disease. Moreover, as more array based CGH datasets become publicly available, meta-analysis studies should further characterize DLBCL subsets. Likewise, given the large collective number of gene expression datasets for DLBCL subtypes and using novel computational methods such as hidden Markov models to predict CNA from gene expression profiling [78] should significantly improve our understanding of the biology, clinical outcome and therapeutic management of DLBCL. Moving forward, microarray analysis should be used in an integrative approach using aCGH, gene expression profiles, SNP arrays and next generation sequencing techniques to better categorize DLBCL subtypes.

In short, our analysis provides a rich starting point for future investigations into the molecular pathogenesis of DLBCL. This review revealed oncogenic pathways that are used differentially by the DLBCL subtypes, reinforcing the view that they represent pathogenetically distinct diseases.

\section{Competing interests}

The authors declare that they have no competing interests.

\section{Authors' contributions}

CAT Lead the whole manuscript writing WC also wrote and did a review of the literature KK did the tables and drafted the first version of the manuscript. RG did the hard work of interpreting the tables and he also created the figures NR revised everything. All authors read and approved the final manuscript.

\section{Author details}

1Department of Pathology \& Laboratory Medicine UCLA - David Geffen UCLA, School of Medicine, Los Angeles, USA. ${ }^{2}$ Ameripath/Quest Diagnostics, Dallas, TX, USA. ${ }^{3}$ Department of Pathology, The UT Southwestern Medical Center, Clinical Cytogenetics, Dallas, USA. ${ }^{4}$ Clinical Cytogenetics, The University of Texas Health Science Center at San Antonio, San Antonio, TX, USA.

Received: 9 April 2012 Accepted: 31 May 2012

Published: 11 September 2012

\section{References}

1. Swerdlow SH, Campo E, Harris NL, Jaffe ES, Pileri SA, Stein H, Thiele J, Vardiman JW: WHO Classification of Tumours of Haematopoietic and Lymphoid Tissues; 2008.

2. Lenz G, et al: Molecular subtypes of diffuse large B-cell lymphoma arise by distinct genetic pathways. Proc Natl Acad Sci U S A 2008, 105(36):13520-13525.

3. Morgensztern D, Govindan R: Is there a role for cetuximab in non small cell lung cancer? Clin Cancer Res 2007, 13(15 Pt 2):4602-4605.

4. Steven S, Nancy Lee H, Elaine J, Harald S, Jurgen T, James V, A. Pileri S, C E: WHO Classification of Tumours of Haematopoietic and Lymphoid Tissues; 2008.

5. Jacobs $\mathrm{G}$, et al: Polymorphisms in the 3 '-untranslated region of the $\mathrm{CDH} 1$ gene are a risk factor for primary gastric diffuse large B-cell lymphoma. Haematologica 2011, 96(7):987-995.

6. Thunberg $U$, et al: Genetic variation in tumor necrosis factor and risk of diffuse large B-cell lymphoma and follicular lymphoma: differences between subgroups in Swedish patients. Leuk Lymphoma 2010, 51(8):1563-1566. 
7. Worrillow $L$, et al: Polymorphisms in the nucleotide excision repair gene ERCC2/XPD and risk of non-Hodgkin lymphoma. Cancer Epidemio/ 2009, 33(3-4):257-260

8. Wang SS, et al: Polymorphisms in DNA repair and one-carbon metabolism genes and overall survival in diffuse large B-cell lymphoma and follicular lymphoma. Leukemia 2009, 23(3):596-602.

9. Kumar $V$, et al: Common variants on $14 q 32$ and $13 q 12$ are associated with DLBCL susceptibility. J Hum Genet 2011, 56(6):436-439.

10. Gurbuxani S, Anastasi J, Hyjek E: Diffuse large B-cell lymphoma-more than a diffuse collection of large B cells: an entity in search of a meaningful classification. Arch Pathol Lab Med 2009, 133(7):1121-1134.

11. Alizadeh AA, et al: Distinct types of diffuse large B-cell lymphoma identified by gene expression profiling. Nature 2000, 403(6769):503-511.

12. Wright $\mathrm{G}$, et al: $\mathrm{A}$ gene expression-based method to diagnose clinically distinct subgroups of diffuse large B cell lymphoma. Proc Natl Acad Sci U S A 2003, 100(17):9991-9996.

13. Rosenwald $A$, et al: The use of molecular profiling to predict survival after chemotherapy for diffuse large-B-cell lymphoma. N Engl J Med 2002, 346(25):1937-1947.

14. Shipp MA, et al: Diffuse large B-cell lymphoma outcome prediction by gene-expression profiling and supervised machine learning. Nat Med 2002, 8(1):68-74.

15. Kramer $\mathrm{MH}$, et al: Clinical relevance of $\mathrm{BCL} 2, \mathrm{BCL} 6$, and $\mathrm{MYC}$ rearrangements in diffuse large B-cell lymphoma. Blood 1998, 92(9):3152-3162.

16. Davis $R E$, et al: Constitutive nuclear factor kappaB activity is required for survival of activated B cell-like diffuse large B cell lymphoma cells. J Exp Med 2001, 194(12):1861-1874.

17. Lam LT, et al: Small molecule inhibitors of IkappaB kinase are selectively toxic for subgroups of diffuse large B-cell lymphoma defined by gene expression profiling. Clin Cancer Res 2005, 11(1):28-40.

18. Rosenwald A, et al: Molecular diagnosis of primary mediastinal B cell lymphoma identifies a clinically favorable subgroup of diffuse large $B$ cell lymphoma related to Hodgkin lymphoma. J Exp Med 2003, 198(6):851-862.

19. Savage KJ, et al: The molecular signature of mediastinal large B-cell lymphoma differs from that of other diffuse large B-cell lymphomas and shares features with classical Hodgkin lymphoma. Blood 2003, 102(12):3871-3879.

20. Savage KJ: Primary mediastinal large B-cell lymphoma. Oncologist 2006, 11(5):488-495.

21. Bentz $M$, et al: Gain of chromosome arm $9 p$ is characteristic of primary mediastinal B-cell lymphoma (MBL): comprehensive molecular cytogenetic analysis and presentation of a novel MBL cell line. Genes Chromosomes Cancer 2001, 30(4):393-401.

22. Wessendorf $S$, et al: Automated screening for genomic imbalances using matrix-based comparative genomic hybridization. Lab Invest 2002, 82(1):47-60.

23. Tagawa $\mathrm{H}$, et al: Comparison of genome profiles for identification of distinct subgroups of diffuse large B-cell lymphoma. Blood 2005, 106(5):1770-1777.

24. Bea S, et al: Diffuse large B-cell lymphoma subgroups have distinct genetic profiles that influence tumor biology and improve geneexpression-based survival prediction. Blood 2005

106(9):3183-3190

25. Chen $W$, et al: Array comparative genomic hybridization reveals genomic copy number changes associated with outcome in diffuse large B-cell lymphomas. Blood 2006, 107(6):2477-2485.

26. Rinaldi $A$, et al: Comparative genome-wide profiling of post-transplant lymphoproliferative disorders and diffuse large B-cell lymphomas. $\mathrm{Br} J$ Haematol 2006, 134(1):27-36.

27. Kreisel F, et al: High resolution array comparative genomic hybridization identifies copy number alterations in diffuse large B-cell lymphoma that predict response to immuno-chemotherapy. Cancer Genet 2011, 204(3):129-137.

28. Perez-Rosado A, et al: BCL6 represses NFkappaB activity in diffuse large B-cell lymphomas. J Pathol 2008, 214(4):498-507.

29. Muris JJ, et al: Apoptosis resistance and response to chemotherapy in primary nodal diffuse large B-cell lymphoma. Hematol Oncol 2006 24(3):97-104.

30. Lam LT, et al: Cooperative signaling through the signal transducer and activator of transcription 3 and nuclear factor-\{kappa\}B pathways in subtypes of diffuse large B-cell lymphoma. Blood 2008, 111 (7):3701-3713.

31. Niu H, Ye BH, Dalla-Favera R: Antigen receptor signaling induces MAP kinase-mediated phosphorylation and degradation of the BCL-6 transcription factor. Genes Dev 1998, 12(13):1953-1961.

32. Steinbach $D$, et al: $A B C A 3$ as a possible cause of drug resistance in childhood acute myeloid leukemia. Clin Cancer Res 2006, 12(14 Pt 1):4357-4363.

33. Pasqualucci $L$, et al: Hypermutation of multiple proto-oncogenes in B-cell diffuse large-cell lymphomas. Nature 2001, 412(6844):341-346.

34. Shaffer AL, Rosenwald A, Staudt LM: Lymphoid malignancies: the dark side of B-cell differentiation. Nat Rev Immunol 2002, 2(12):920-932.

35. Wlodarska I, et al: FOXP1, a gene highly expressed in a subset of diffuse large B-cell lymphoma, is recurrently targeted by genomic aberrations. Leukemia 2005, 19(8):1299-1305.

36. Motoyama $M$, et al: Positive and negative regulation of nuclear factorkappaB-mediated transcription by IkappaB-zeta, an inducible nuclear protein. J Biol Chem 2005, 280(9):7444-7451.

37. Lenz $\mathrm{G}$, et al: Aberrant immunoglobulin class switch recombination and switch translocations in activated B cell-like diffuse large B cell lymphoma. J Exp Med 2007, 204(3):633-643.

38. O'Donnell KA, et al: c-Myc-regulated microRNAs modulate E2F1 expression. Nature 2005, 435(7043):839-843.

39. Palanisamy $\mathrm{N}$, et al: Similar patterns of genomic alterations characterize primary mediastinal large-B-cell lymphoma and diffuse large-B-cell lymphoma. Genes Chromosomes Cancer 2002, 33(2):114-122.

40. Green MR, et al: Integrative analysis reveals selective 9p24.1 amplification, increased PD-1 ligand expression, and further induction via JAK2 in nodular sclerosing Hodgkin lymphoma and primary mediastinal large B-cell lymphoma. Blood 2010, 116(17):77-3268.

41. Joos $\mathrm{S}$, et al: Primary mediastinal (thymic) B-cell lymphoma is characterized by gains of chromosomal material including $9 p$ and amplification of the REL gene. Blood 1996, 87(4):1571-1578.

42. Barth TF, et al: Gains of $2 p$ involving the REL locus correlate with nuclear c-Rel protein accumulation in neoplastic cells of classical Hodgkin lymphoma. Blood 2003, 101(9):3681-3686.

43. Wessendorf $\mathrm{S}$, et al: Further delineation of chromosomal consensus regions in primary mediastinal B-cell lymphomas: an analysis of 37 tumor samples using high-resolution genomic profiling (array-CGH). Leukemia 2007, 21(12):2463-2469.

44. Said J: Diffuse aggressive B-cell lymphomas. Adv Anat Pathol 2009, 16(4):216-235

45. Feys $\mathrm{T}$, et al: A detailed inventory of DNA copy number alterations in four commonly used Hodgkin's lymphoma cell lines. Haematologica 2007, 92(7):913-920.

46. Heyning $\mathrm{FH}$, et al: Array-based comparative genomic hybridisation analysis reveals recurrent chromosomal alterations in primary diffuse large B cell lymphoma of bone. J Clin Pathol 2010, 63(12):1095-1100.

47. Booman $M$, et al: Genomic alterations and gene expression in primary diffuse large B-cell lymphomas of immune-privileged sites: the importance of apoptosis and immunomodulatory pathways. J Pathol 2008, 216(2):209-217

48. Montesinos-Rongen M, Siebert $R$, Deckert M: Primary lymphoma of the central nervous system: just DLBCL or not? Blood 2009, 113(1):7-10.

49. Camilleri-Broet $\mathrm{S}$, et al: A uniform activated B-cell-like immunophenotype might explain the poor prognosis of primary central nervous system lymphomas: analysis of 83 cases. Blood 2006, 107(1):190-196.

50. Belaud-Rotureau MA, et al: Inactivation of p16INK4a/CDKN2A gene may be a diagnostic feature of large $B$ cell lymphoma leg type among cutaneous B cell lymphomas. Virchows Arch 2008, 452(6):607-620.

51. Dijkman $\mathrm{R}$, et al: Array-based comparative genomic hybridization analysis reveals recurrent chromosomal alterations and prognostic parameters in primary cutaneous large B-cell lymphoma. J Clin Oncol 2006, 24(2):296-305

52. Franke $S$, et al: Comparative genomic hybridization pattern distinguishes T-cell/histiocyte-rich B-cell lymphoma from nodular lymphocyte predominance Hodgkin's lymphoma. Am J Pathol 2002, 161(5):1861-1867.

53. Monti $\mathrm{S}$, et al: Molecular profiling of diffuse large B-cell lymphoma identifies robust subtypes including one characterized by host inflammatory response. Blood 2005, 105(5):1851-1861. 
54. Matolcsy A, Chadburn A, Knowles DM: De novo CD5-positive and Richter's syndrome-associated diffuse large B cell lymphomas are genotypically distinct. Am J Pathol 1995, 147(1):207-216.

55. Harada $\mathrm{S}$, et al: Molecular and immunological dissection of diffuse large B cell lymphoma: CD5+, and CD5- with CD10+ groups may constitute clinically relevant subtypes. Leukemia 1999, 13(9):1441-1447.

56. Yamaguchi $M$, et al: De novo CD5+ diffuse large B-cell lymphoma: a clinicopathologic study of 109 patients. Blood 2002, 99(3):815-821.

57. Tagawa $\mathrm{H}$, et al: Genome-wide array-based comparative genomic hybridization of diffuse large B-cell lymphoma: comparison between CD5-positive and CD5-negative cases. Cancer Res 2004, 64(17):5948-5955.

58. Miwa $\mathrm{H}$, et al: DNA sequences of the immunoglobulin heavy chain variable region gene in pyothorax-associated lymphoma. Oncology 2002, 62(3):241-250.

59. Hongyo $T$, et al: Frequent p53 mutations at dipyrimidine sites in patients with pyothorax-associated lymphoma. Cancer Res 1998, 58(6):1105-1107.

60. Yamato $\mathrm{H}$, et al: Evidence for local immunosuppression and demonstration of c-myc amplification in pyothorax-associated lymphoma. Histopathology 2001, 39(2):163-171.

61. Nishiu $M$, et al: Distinct pattern of gene expression in pyothoraxassociated lymphoma (PAL), a lymphoma developing in long-standing inflammation. Cancer Sci 2004, 95(10):828-834.

62. Takakuwa T, et al: Establishment and characterization of unique cell lines derived from pyothorax-associated lymphoma which develops in longstanding pyothorax and is strongly associated with Epstein-Barr virus infection. Cancer Sci 2003, 94(10):858-863.

63. Chang CC, et al: Genomic profiling of plasmablastic lymphoma using array comparative genomic hybridization $(\mathrm{aCGH})$ : revealing significant overlapping genomic lesions with diffuse large B-cell lymphoma. J Hematol Oncol 2009, 2:47.

64. Colomo L, et al: Diffuse large B-cell lymphomas with plasmablastic differentiation represent a heterogeneous group of disease entities. Am J Surg Pathol 2004, 28(6):736-747.

65. Du MQ, Bacon CM, Isaacson PG: Kaposi sarcoma-associated herpesvirus/ human herpesvirus 8 and lymphoproliferative disorders. J Clin Pathol 2007, 60(12):1350-1357.

66. Cesarman E, Mesri EA: Kaposi sarcoma-associated herpesvirus and other viruses in human lymphomagenesis. Curr Top Microbiol Immunol 2007, 312:263-287.

67. Luan SL, et al: Primary effusion lymphoma: genomic profiling revealed amplification of SELPLG and CORO1C encoding for proteins important for cell migration. J Pathol 2010, 222(2):166-179.

68. Ohshima K, et al: Chromosomal and comparative genomic analyses of HHV-8-negative primary effusion lymphoma in five HIV-negative Japanese patients. Leuk Lymphoma 2002, 43(3):595-601.

69. Hummel $M$, et al: A biologic definition of Burkitt's lymphoma from transcriptional and genomic profiling. N Engl J Med 2006, 354(23):2419-2430.

70. McClure RF, et al: Adult B-cell lymphomas with burkitt-like morphology are phenotypically and genotypically heterogeneous with aggressive clinical behavior. Am J Surg Pathol 2005, 29(12):1652-1660.

71. Ferreri AJ, et al: Anthracycline-based chemotherapy as primary treatment for intravascular lymphoma. Ann Oncol 2004, 15(8):1215-1221.

72. Reichard KK, McKenna RW, Kroft SH: ALK-positive diffuse large B-cell lymphoma: report of four cases and review of the literature. Mod Pathol 2007, 20(3):310-319.

73. Shimoyama $Y$, et al: Senile Epstein-Barr virus-associated B-cell lymphoproliferative disorders: a mini review. J Clin Exp Hematop 2006, 46(1):1-4.

74. Oyama T, et al: Senile EBV+B-cell lymphoproliferative disorders: a clinicopathologic study of 22 patients. Am J Surg Pathol 2003, 27(1):16-26

75. Ohno $\mathrm{H}$, et al: Epstein-Barr virus-positive diffuse large B-cell lymphoma carrying a t(9;14)(p13;q32) translocation. Int J Hematol 2009, 89(5):8-704.

76. Du MQ, et al: Kaposi sarcoma-associated herpesvirus infects monotypic (IgM lambda) but polyclonal naive B cells in Castleman disease and associated lymphoproliferative disorders. Blood 2001, 97(7):6-2130.

77. Godde-Salz E, Feller AC, Lennert K: Chromosomal abnormalities in lymphogranulomatosis $\mathrm{X}(\mathrm{LgrX})$ /angioimmunoblastic lymphadenopathy (AILD). Leuk Res 1987, 11(2)):90-181.
78. Geng H, et al: Virtual CGH: an integrative approach to predict genetic abnormalities from gene expression microarray data applied in lymphoma. BMC Med Genomics 2011, 4:32.

doi:10.1186/1756-8722-5-54

Cite this article as: Tirado et al.: Genomic profiling using array comparative genomic hybridization define distinct subtypes of diffuse large b-cell lymphoma: a review of the literature. Journal of Hematology \& Oncology 2012 5:54

\section{Submit your next manuscript to BioMed Central and take full advantage of:}

- Convenient online submission

- Thorough peer review

- No space constraints or color figure charges

- Immediate publication on acceptance

- Inclusion in PubMed, CAS, Scopus and Google Scholar

- Research which is freely available for redistribution

Submit your manuscript at www.biomedcentral.com/submit
C Biomed Central 